\title{
Radio Insight into the Nature of Type IIb Progenitors
}

\author{
Christopher J. Stockdale ${ }^{1}$, S. D. Ryder ${ }^{2}$, A. Horesh ${ }^{3}$, K. W. Weiler ${ }^{4}$, \\ N. Panagia ${ }^{5}$, S. D. Van Dyk ${ }^{6}$, F. E. Bauer ${ }^{7}$, S. Immler ${ }^{8}$, \\ R. A. Sramek ${ }^{9}$, D. Pooley ${ }^{10}$, J. M. Marcaide ${ }^{11}$, and N. Kassim ${ }^{12}$ \\ ${ }^{1}$ Marquette University \\ Dept. of Physics, P.O. Box 1811, Milwaukee, WI 53201, USA \\ email: chris.stockdale@mu.edu \\ ${ }^{2}$ Australian Astronomical Observatory \\ P.O. Box 296, Epping, NSW 1710, Australia \\ ${ }^{3}$ California Institute of Technology \\ Cahill Center for Astrophysics, Pasadena, CA, 91125, USA \\ ${ }^{4}$ Computational Physics, Inc. \\ 8001 Braddock Rd., Suite 210, Springfield, VA 22151, USA \\ ${ }^{5}$ Space Telescope Science Institute \\ 3700 San Martin Drive, Baltimore, MD 21218, USA \\ ${ }^{6}$ Spitzer Science Center/Caltech \\ Mailcode 220-6, Pasadena, CA 91125, USA \\ ${ }^{7}$ Pontificia Universidad Católica de Chile \\ Departamento de Astronomía y Astrofísica, Casilla 306, Santiago 22, Chile \\ ${ }^{8}$ Center for Research and Exploration in Space Science and Technology \\ NASA Goddard Space Flight Center, Greenbelt, MD 20771, USA \\ ${ }^{9}$ National Radio Astronomy Observatory \\ P.O. Box O, Socorro, NM 87801 USA \\ ${ }^{10}$ Sam Houston State University \\ Department of Physics, Farrington Building, Suite 204, Box 2267, Huntsville, TX 77341 USA \\ ${ }^{11}$ Universidad de Valencia \\ Departamento de Astronomía, 46100 Burjassot, Spain \\ ${ }^{12}$ Naval Research Laboratory \\ Code 7210, Washington, D.C. 20375, USA
}

\begin{abstract}
We present the results of over two decades of radio observations of type IIb Supernovae with the Very Large Array and the Australia Telescope Compact Array. These radio studies illustrate the need for multi-wavelength follow-up to determine the progenitor scenario for type IIb events.
\end{abstract}

\section{Overview}

Type IIb Supernovae often have very similar optical spectra initially and frequently evolve into type Ib Supernovae. The optical classification IIb was invented to describe the spectral characteristics of type II SN 1987K, distinguished by its weak hydrogen absorption lines and a weak HeI emission line (Filippenko 1988). The recent SNe 2011dh (Horesh et al. 2011a; Horesh et al. 2011b; Soderberg et al. 2011; Krauss et al. 2012) and 2011hs (Ryder et al. 2011) bring the total type IIb SNe detected in the radio to eight, including SNe 1993J (Weiler et al. 2007), 2001gd (Stockdale et al. 2007), 2001ig (Ryder 
et al. 2004), 2003bg (Soderberg et al. 2006), 2008ax (Stockdale et al. 2008a; Roming et al. 2009), and 2008bo (Stockdale et al. 2008b).

SN 1993J is the best studied case of a radio-loud supernova, (Weiler et al. 2007; MartiVidal et al. 2011; and references therein) reaching maximum radio light at $6 \mathrm{~cm}$ near 150 days after explosion and declining with a power-law time decay index of -0.73 and a spectral index of -0.81. SN 2001gd had a similar radio evolution to SN 1993J with both SNe showing no clear signs of short-term variability (Stockdale et al. 2007). However both show unexpected declines in their radio light curves that are interpreted as abrupt changes in the mass-loss rates of their progenitors. For SN 1993J, this change implies that its progenitor star underwent an abrupt increase in its mass-loss rate from $3 \times 10^{-6}$ up to $7 \times 10^{-6}$ solar masses $\mathrm{yr}^{-1}$ between 10,000 and 7,000 yrs prior to explosion, with a nearly linear decline in mass-loss rate to $\sim 10^{-6}$ solar masses $\mathrm{yr}^{-1}$ just prior to explosion.

Radio emission from another excellent example of this type IIb class, SN 2001ig, shows a periodic oscillation in the radio light curve of 150 days, while SN 2008ax exhibits an approximate periodicity of 80 days in its radio emission. The periodicity observed in SNe 2001ig (Ryder et al. 2004), 2003bg (Soderberg et al. 2006) and 2008ax (Roming et al. 2009) can be explained by a binary companion that influenced the wind-established CSM of the SN progenitor star to create spiral density fluctuations that the SN blastwave later shocks (e.g. SN 1979C Weiler et al. 1992; Schwarz \& Pringle 1996).

\section{Implications}

These studies demonstrate that the radio properties can effectively allow us to distinguish between two possible progenitor scenarios which have nearly indistinguishable optical spectra: 1) a single massive star which has shed most of its hydrogen as a function of normal evolution and 2) a less massive star whose hydrogen envelope is being influenced and depleted by a binary companion. The prevailing evidence appears to lean toward most type IIb SNe being binary systems with varying degrees of interaction (Van Dyk et al. 2011; Chevalier \& Soderberg 2010).

\section{References}

Chevalier \& Soderberg 2010, ApJ, 711, L40

Horesh, A. et al. 2011, ATEL, 3405

Horesh, A. et al. 2011, ATEL, 3411

Filippenko, A. 1988, AJ 96, 1941

Krauss, M., et al. 2012, arXiv, 1201.0770

Marti-Vidal et al. 2011, A\& A, 526A, 143

Roming, P., et al. 2009, ApJ, 704, L118

Ryder, S. D., et al. 2004, MNRAS, 349, 1093

Ryder, S. D. et al. 2011, ATEL, 3789

Schwarz \& Pringle 1996, MNRAS, 282, 1018

Soderberg, A. M., et al. 2006, ApJ, 651, 1005

Soderberg, A. M., et al. 2011, arXiv, 1107.1876

Stockdale, C. J., et al. 2007, ApJ, 671, 689

Stockdale, C. J., et al. 2008, CBET, 1299

Stockdale, C. J., et al. 2008, IAUC, 8939

Van Dyk, S. D., et al. 2011, ApJ, 741, L28

Weiler, K. W., et al. 2007, ApJ, 671, 1959

Weiler et al. 1992, ApJ, 399, 672 